\title{
Pregnancy-induced Progression of Keratoconus in a 37-Year-Old Patient
}

\author{
Juliana Glicéria, Bruno Freitas Valbon, Rodrigo Teixeira Santos, Renato Ambrósio Jr
}

\section{ABSTRACT}

Purpose: To report a case of keratoconus that progressed during pregnancy without any accompanying disease.

Methods: A case report.

Results: A patient with keratoconus that worsened during pregnancy demonstrates that pregnancy can be a previously unrecognized risk factor for progression of keratoconus.

Conclusion: To our knowledge, this is the first case reported in Brazil and the third paper in the world showing pregnancyinduced keratoconus progression in a patient with no accompanying disease.

Keywords: Keratoconus, Corneal ectasia, Pregnancy, Risk factors, Case report.

How to cite this article: Glicéria J, Valbon BF, Santos RT, Ambrósio R Jr. Pregnancy-induced Progression of Keratoconus in a 37-Year-Old Patient. Int J Kerat Ect Cor Dis 2013;2(2):84-88.

\section{Source of support: Nil}

Conflict of interest: None declared

\section{INTRODUCTION}

Keratoconus is characterized by progressive corneal ectasia and estromal thinning, increased myopia and irregular astigmatism. ${ }^{1}$ The pathological process underlying keratoconus have not been fully understood, but is accepted that it is a clinical condition with multifactorial etiology that ends in corneal conical protrusion. Many factors can aggravate keratoconus progression such as eye rubbing. ${ }^{2}$

Recently, the effect of pregnancy on the progression of an ectatic condition has been reported. In the literature, four cases of keratoconus progression were reported as developing during pregnancy without any accompanying factors.

This report provides adds to this body of literature with the case of a 37-year-old patient that had stabilized disease for over 10 years.

\section{CASE REPORT}

A 37-year-old woman was first seen in the clinic in November 2007 for a second opinion. She had been previously diagnosed as having keratoconus and had presented a relatively stable ectasia in the left eye (OS) for over ten years. She complained of progressive loss of corrected visual acuity during pregnancy that had not improved four after delivery. Her previous medical history consisted of a lamellar keratoplasty in the right eye (OD) in 2000 and no other comorbidities. There was no family history of keratoconus or corneal transplant, no sporadic eye rubbing, and no known atopic diseases in the patient or her family.

In November 2007, her best corrected visual acuity was $20 / 30$ in the both eyes with her current spectacles. The biomicroscopic examination showed a clear corneal transplant in OD and a transparent cornea in OS with thickened nerves and few papillae. ORA (Ocular Response Analyser) showed a Goldmann correlated intraocular pressure (IOPg) of $2.5 \mathrm{~mm} \mathrm{Hg}$, a corneal compensated intraocular pressure (IOPcc) of $8.4 \mathrm{~mm} \mathrm{Hg}$, a corneal hysteresis $(\mathrm{CH})$ of 7.1 and a corneal resistance factor (CRF) of 3.7 in OS. Corneal topography measured by front sagital curvature in Pentacam (Oculus Optikgeräte GmBH, Wetzlar, Germany) revealed a maximum keratometric power of 63.9 D. The thickness at the thinnest point evaluated was $339 \mu \mathrm{m}$ in the left eye. We observed from the anterior corneal sagital curvature map a simulated keratometry of $53.1 \times 57.0$ @169.8 (Figs 1A and B).

In January 2013, the patient returned complaining of worse vision in OS. Best corrected visual acuity was 20/30 in OD and 20/40 in OS. The biomicroscopic examination showed no significant changes. ORA showed an IOPg of $1.7 \mathrm{~mm} \mathrm{Hg}$, IOPcc of $9.0 \mathrm{~mm} \mathrm{Hg}, \mathrm{CH}$ of 5.9 and CRF of 2.4 , indicating a weaker cornea in OS. We repeated the tomography examination using the Pentacam which showed a maximum keratometric power of $68.5 \mathrm{D}$ and a thinnest point of $340 \mu \mathrm{m}$ in OS. At the anterior corneal sagittal curvature map, we could see an increase in simulated keratometry to $58.0 \times 63.0 @ 170.1 \mathrm{D}$ (Figs 1A and B). In front and back elevation maps, we can observe the worsening in corneal protrusion when the maps are compared (Figs $2 \mathrm{~A}$ and $\mathrm{B}$ ). These data indicate the deteriorating state of keratoconus in OS.

The Corvis ST (Scheimpflug Technology recently introduced by Oculus Optikgeräte $\mathrm{GmbH}$, Wetzlar, Germany) demonstrated a deformation's amplitude of $1.69 \mathrm{~mm}$ (Fig. 3). Ambrosio et $\mathrm{al}^{3}$ reported observed differences in the combined parameters based on a linear regression analysis of data from the Corvis ST for four study groups (i.e. normal, forme frustre keratoconus, asymmetric bow tie, and keratoconus). The mean deformation amplitude was $1.02 \pm 0.09 \mathrm{~mm}$ (i.e. the range was: 0.78 to 1.26 ) in the normal eyes reviewed in this article. 


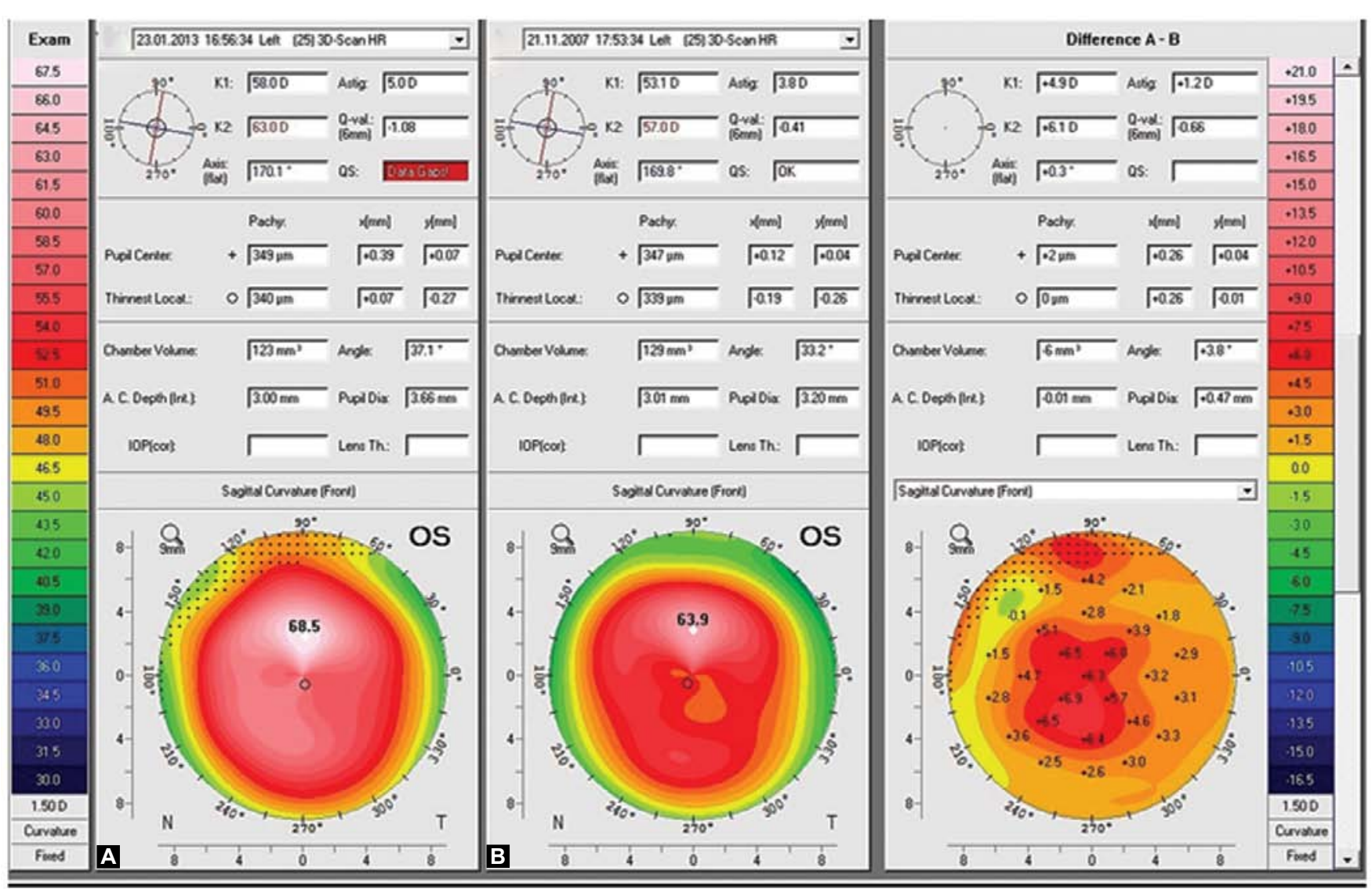

Figs 1A and B: Evaluation of corneal topographical (front sagittal curvatures maps): after pregnancy (A) and before pregnancy (B) and the differential map. Pentacam (Oculus Optikgeräte $\mathrm{GmBH}$, Wetzlar, Germany)

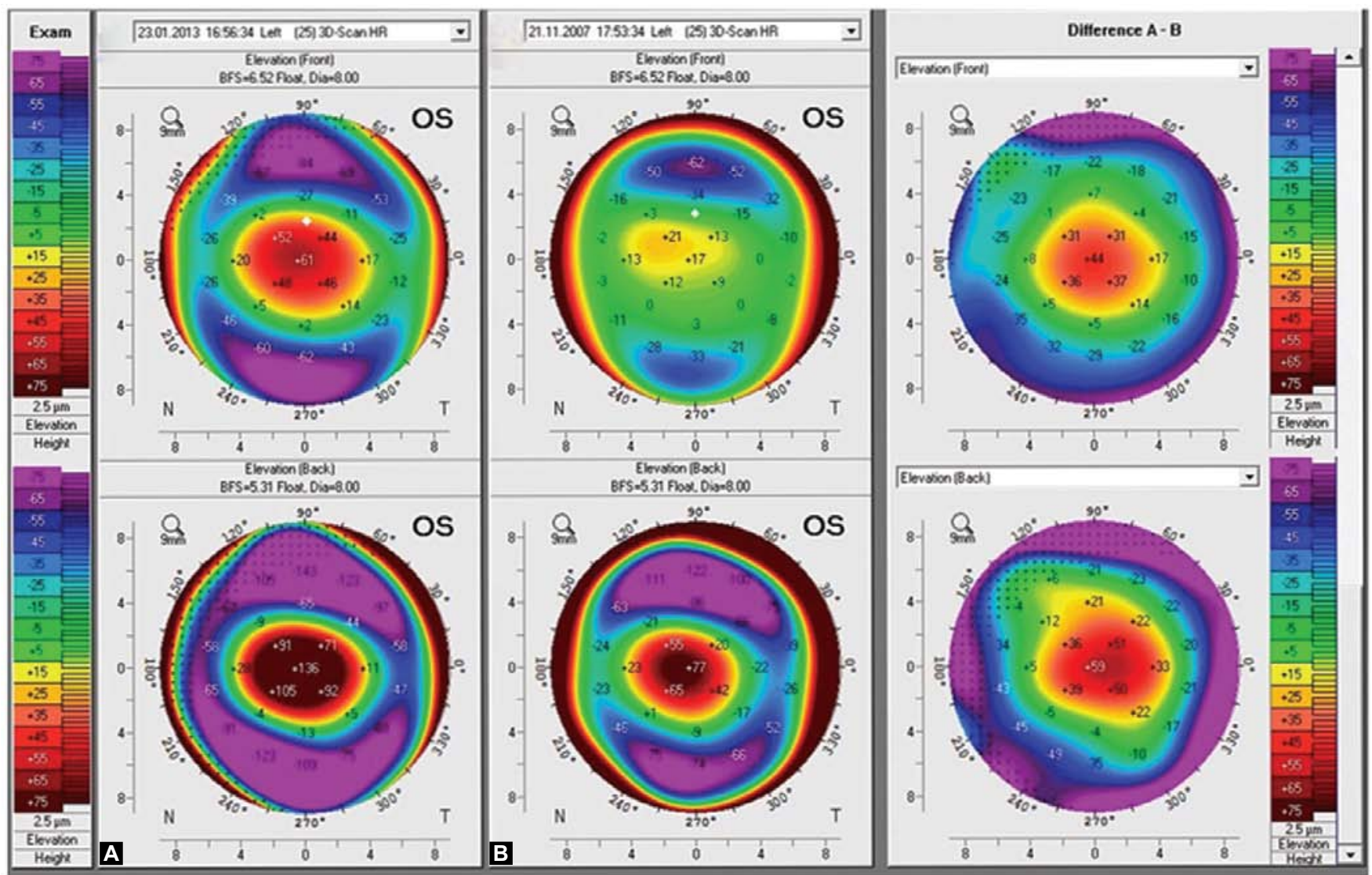

Figs 2A and B: Evaluation of corneal elevation (front and back elevation maps): after pregnancy (A) and before pregnancy (B) and the differential map. Pentacam (Oculus Optikgeräte $\mathrm{GmBH}$, Wetzlar, Germany) 


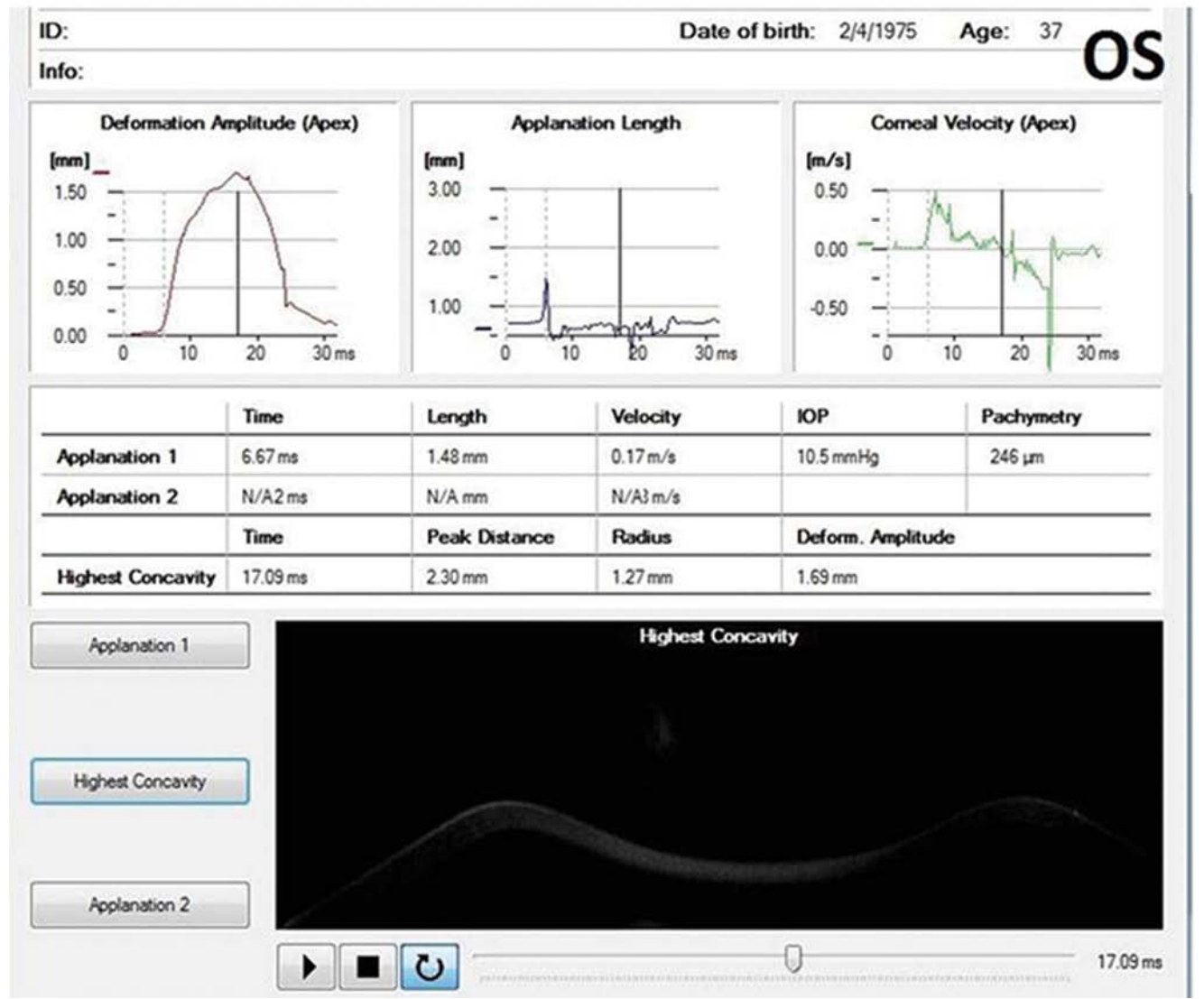

Fig. 3: Corneal biomechanical evaluation: increased deformation amplitude index $(1.69 \mathrm{~mm})$ demonstrates the cornea's weakness in keratoconus. Corvis (Oculus Optikgeräte GmbH, Wetzlar, Germany)

Considering the treatments available, keraring and corneal cross-linking were recommended to this patient to regularize and strengthen the cornea.

\section{DISCUSSION}

In this case, the patient fulfilled two of the criteria that attest to the progression of keratoconus: an increase in one or more diopters in the mean keratometry and an increase of at least 1.0 diopter in astigmatism or an increase of at least 0.5 diopter in manifest spherical equivalent refraction, ${ }^{2}$ confirming that keratoconus was worsening and that precocious intervention was necessary.

In 2010, the Collaborative Longitudinal Evaluation of Keratoconus Study reported the influence of hormone status on the severity and progression of keratoconus involving patients between 48 and 59 years old to evaluate the effects of menopause and use of external hormonal replacement therapy. ${ }^{4}$ That study could not detect any influence of hormone status on keratoconus patients. However, they did not include any pregnant patients in that study. The group admitted that the slow progression of keratoconus in that particular age group studied represented a limitation.

Gatzioufas and Thanos ${ }^{5}$ have reported a case of hypothyroxinemia-induced acute corneal hydrops during pregnancy. The authors suggested that the progression was related to thyroid gland dysfunction because the deterioration of keratoconus coincided with the lowest plasma thyroxin level. Hafezi and Iseli ${ }^{6}$ reported a case of bilateral iatrogenic keratectasia 26 months after LASIK, which developed during pregnancy.

Our patient, contrary to previous studies, had no associated disease or predisposing factor. Similar to our case, Bilgihan et al ${ }^{1}$ reported four cases of keratoconus without any accompanying disease that progressed during pregnancy.

Recent studies have suggested that changes in estrogen levels may play a role in corneal ectasia. ${ }^{7,8}$ Estrogen receptors have been identified in cornea, ${ }^{9-11}$ and a significant stiffness-reducing effect of estrogen on cornea has been shown in an experimental study. These findings suggest that high estrogen- states, like pregnancy, may predispose biomechanically weak corneas to ectasia development or progression. $^{12}$

Various studies have demonstrated elevated levels of collagenolytic and gelatinolytic activities in keratoconic corneas. ${ }^{13-17}$ Matrix metalloproteinase (MMP) levels are increased, whereas tissue inhibitors of MMPs (TIMPs) are decreased in the keratoconic corneas ${ }^{17-19}$ as well as during pregnancy too. ${ }^{20-23}$ It has been suggested that proteinases 
(collagenases and MMPs) may play an important role in the pathogenesis of keratoconus. ${ }^{24}$ Furthermore, proteinase enzymes play key roles in various points of pregnancy. These enzymes contribute to the extracellular matrix remodeling required for follicular development and ovulation, for loosening of the collagen framework of the endometrium for implantation, growth and softening of the cervix, and for relaxation of the pelvic bones for delivery. ${ }^{25-28}$

It therefore seems clear that increased levels of proteolytic enzymes and decreased levels of their inhibitors during pregnancy are important for the progression of keratoconus.

An important molecule that plays key roles in pregnancy is relaxin. Relaxin was discovered in 1926 by Hisaw, ${ }^{29}$ who noted relaxation of the pubic ligament in virgin guinea pigs after injection of serum from pregnant guinea pigs. The most consistent biological effect of relaxin is its ability to stimulate the breakdown of collagen. It increases the synthesis of the collagen-degrading enzymes and MMPs and decreases the synthesis of TIMPs. ${ }^{30}$ Therefore; relaxin may also play a role in the progression of keratoconus during pregnancy through MMPs and TIMPs.

Hormonal changes during pregnancy may affect corneal biomechanics negatively. They are associated with increased corneal thickness, decreased corneal hysteresis and decreased corneal resistant factor. Therefore, pregnancy can be a previously unrecognized risk factor for progression of keratoconus.

If further studies demonstrate a significant risk of keratoconus progression during pregnancy, then corneal cross-linking should be more extensively applied in patients with keratoconus that are considering becoming pregnant. Thus, more studies are required to elucidate the clinical correlation of keratoconus and pregnancy.

\section{REFERENCES}

1. Bilgihan K, Hondur A, Sul S, Ozturk S. Pregnancy-induced progression of keratoconous. Cornea 2011 Sep;30(9):991-994.

2. Rabinowitz YS. Keratoconus. Surv Ophthalmol 1998;42(4):297319.

3. Ambrosio R Jr. Corneal biomechanical assessment using dynamic Ultra-High-Speed Scheimpflug Technology Noncontact tonometry (UHS-ST NCT): Preliminary Results. Symposium and congress of the American Society of Cataract and Refractive Surgery. 2001. Poster. San Diego, USA.

4. Fink BA, Sinnott LT, Wagner H, et al. CLEK Study Group. The influence of gender and hormone status on the severity and progression of keratoconus. Cornea 2010;29:65-72.

5. Gatzioufas Z, Thanos S. Acute keratoconus induced by hypothyroxinemia during pregnancy. J Endocrinol Invest 2008; 31:262-266.
6. Hafezi F, Iseli HP. Pregnancy-related exacerbation of iatrogenic keratectasia despite corneal collagen crosslinking. J Cataract Refract Surg 2008;34:1219-1221

7. Sunness JS. The pregnant woman's eye. Surv Ophthalmol 1988; 32(4):219-238.

8. Wienreb RN, Lu A, Beeson C. Maternal corneal thickness during pregnancy. Am J Ophthalmol 1988;105(3):258-260.

9. Suzuki T, Kinoshita Y, Tachibana M, et al. Expression of sex steroid hormone receptors in human cornea. Curr Eye Res 2001; 22:28-33.

10. Hadeyama T, Nakayasu K, Ha NT, et al. Expression of estrogen receptors alpha and beta, androgen receptors and progesterone receptors in human cornea. Nippon Ganka Gakkai Zasshi 2002; 106:557-564

11. Suzuki T, Richards SM, Liu S, et al. Influence of sex on gene expression in human corneal epithelial cells. Mol Vis 2009; 15:2554-2569.

12. Spoerl E, Zubaty V, Raiskup-Wolf F, et al. Estrogen-induced changes in biomechanics in the cornea as a possible reason for keratectasia. Br J Ophthalmol 2007;91:1547-1550.

13. Kao WW, Vergnes JP, Ebert J, et al. Increased collagenase and gelatinase activities in keratoconus. Biochem Biophys Res Commun. 1982;107:929-936.

14. Rehany U, Lahav M, Shoshan S. Collagenolytic activity in keratoconus. Ann Ophthalmol 1982;14:751-754.

15. Kenney MC, Chwa M, Escobar M, et al. Altered gelatinolytic activity by keratoconus corneal cells. Biochem Biophys Res Commun 1989;161:353-357.

16. Mackiewicz Z, Määttä M, Stenman $M$, et al. Collagenolytic proteinases in keratoconus. Cornea 2006;25:603-610.

17. Zhou L, Sawaguchi S, Twining SS, et al. Expression of degradative enzymes and protease inhibitors in corneas with keratoconus. Invest Ophthalmol Vis Sci 1998;39:1117-1124.

18. Kenney MC, Chwa M, Opbroek AJ, et al. Increased gelatinolytic activity in keratoconus keratocyte cultures. A correlation to an altered matrix metalloproteinase-2/tissue inhibitor of metalloproteinase ratio. Cornea 1994;13:114-124.

19. Smith VA, Matthews FJ, Majid MA, et al. Keratoconus: Matrix metalloproteinase-2 activation and TIMP modulation. Biochim Biophys Acta 2006;1762:431-439.

20. Morrison JJ, Clark IM, Powell EK, et al. Tissue collagenase: serum levels during pregnancy and parturition. Eur J Obstet Gynecol Reprod Biol 1994;54:71-75.

21. Granström LM, Ekman GE, Malmström A, et al. Serum collagenase levels in relation to the state of the human cervix during pregnancy and labor. Am J Obstet Gynecol. 1992;167:1284-1288.

22. Clark IM, Morrison JJ, Hackett GA, et al. Tissue inhibitor of metalloproteinases: serum levels during pregnancy and labor, term and preterm. Obstet Gynecol 1994;83:532-537.

23. Osmers R, Tschesche H, Rath W, et al. Serum collagenase levels during pregnancy and parturition. Eur J Obstet Gynecol Reprod Biol 1994;53:55-57.

24. Balasubramanian SA, Pye DC, Willcox MD. Are proteinases the reason for keratoconus? Curr Eye Res 2010;35:185-191.

25. Huang SC, Sheu BC, Chang WC, et al. Extracellular matrix proteases - cytokine regulation role in cancer and pregnancy. Front Biosci 2009;14:1571-1588.

26. Hwang JJ, Lin SW, Teng CH, et al. Relaxin modulates the ovulatory process and increases secretion of different gelatinases from granulosa and theca-interstitial cells in rats. Biol Reprod 1996;55:1276-1283. 
27. Rajabi MR, Solomon S, Poole AR. Hormonal regulation of interstitial collagenase in the uterine cervix of the pregnant guinea pig. Endocrinology 1991;128:863-871.

28. Rajabi MR, Solomon S, Poole AR. Biochemical evidence of collagenases mediated collagenolysis as a mechanism of cervical dilatation at parturition in the guinea pig. Biol Reprod 1991; 45:764-772.

29. Hisaw FL. Experimental relaxation of the pubic ligament of the guinea pig. Proc Soc Exp Biol Med 1926;23:661-663.

30. Sherwood OD. Relaxin's physiological roles and other diverse actions. Endocr Rev 2004;25:205-234.

\section{ABOUT THE AUTHORS}

\section{Juliana Glicéria (Corresponding Author)}

Resident, Department of Ophthalmology, Hospital da Piedade (RJ); Research Associate, Rio de Janeiro Corneal Tomography and
Biomechanics Study Group - Rio de Janeior, RJ, Brazil, Phone: 552125971132, e-mail: jugli@globo.com

\section{Bruno Freitas Valbon}

Postgraduate Student, Department of Ophthalmology, University of São Paulo, São Paulo, Brazil

\section{Rodrigo Teixeira Santos}

Fellow, Department of Ophthalmology, Federal University of São Paulo, São Paulo, Brazil

\section{Renato Ambrósio Jr}

Founder and Director, Rio de Janeiro Corneal, Tomography and Biomechanics Study Group; Director of Refractive Surgery of Inst. Olhos R Ambrosio and VisareRIO, Rio de Janeiro, RJ, Brazil; Associate Professor, Department of Ophthalmology, Federal University of São Paulo, São Paulo, Brazil 